Article

\title{
Nanofiltration of Succinic Acid in Strong Alkaline Conditions
}

\author{
Klaus Schlackl ${ }^{1, *}$, Richard Herchl ${ }^{2}$ and Wolfgang Samhaber ${ }^{3}$ \\ 1 Kompetenzzentrum Holz GmbH, 4040 Linz, Austria \\ 2 Lenzing AG, 4860 Lenzing, Austria; r.herchl@lenzing.com \\ 3 Department of Process Engineering, Johannes Kepler University, 4040 Linz, Austria; \\ wolfgang.samhaber@jku.at \\ * Correspondence: k.schlackl@wood-kplus.at; Tel.: +43-7672-701-2088
}

Received: 8 October 2019; Accepted: 7 November 2019; Published: 8 November 2019

check for updates

\begin{abstract}
Nanofiltration is considered to be an appropriate separation technique in the production of bio-based materials. For the utilization of process streams from the viscose-fiber production, understanding the separation behavior of organic compounds in highly alkaline solutions is necessary. Experiments with succinic acid in sodium hydroxide $(\mathrm{NaOH})$ solutions with varying concentrations up to $5 \mathrm{~mol} \mathrm{~L}^{-1}$ were performed with the NP030 membrane from Microdyn Nadir. Furthermore, experiments with aqueous disodium succinate and solutions of sodium sulfate in sodium hydroxide were carried out. The influence of concentration ratios and temperature was studied. The Spiegler and Kedem model as well as the Pusch model were applied to fit the experimental data. Additionally, scanning electron microscopy (SEM) and infrared (ATR-IR) measurements were performed to validate the chemical and thermomechanical stability of the membrane. The succinic acid retention varies with its degree of dissociation. In a fully dissociated form, the $\mathrm{NaOH}$ concentration shows no impact on the retention. In contrast, the retention of sulfate decreases with increasing $\mathrm{NaOH}$ concentration.
\end{abstract}

Keywords: nanofiltration; $\mathrm{NaOH}$; succinic acid; succinate; reflection coefficient

\section{Introduction}

Nanofiltration has the potential to be a highly effective separation method for the utilization of viscose-fiber production side streams. Furthermore, it can be used as a downstream process for the purification of process chemicals. A possible application is the removal of hemi celluloses and other wood degradation products from the highly caustic steeping lye. Hence, the lye could be recycled to the process and the demand of the make-up caustic solution can be significantly reduced. The total organic carbon (TOC) content can basically be divided into two fractions. The xylose oligo- and polymers with a molecular weight from $\sim 600$ to $\sim 50,000 \mathrm{~g} \mathrm{~mol}^{-1}$ (beta fraction) and the hydroxyl acids, ranging from $\mathrm{C}_{2}$ up to $\mathrm{C}_{6}$ acids (gamma fraction).

The application of ceramic ultrafiltration membranes for the hemi cellulose removal (feed concentration: $30 \mathrm{~g} \mathrm{~L}^{-1}$ ) from steeping lye containing 18\% sodium hydroxide was tested by Saurabh [1]. The used membranes had a molecular weight cut of (MWCO) of 3, 5, and $15 \mathrm{kDa}$. The membranes showed maximum retention of $0.68(\mathrm{MWCO}=3 \mathrm{kDa}), 0.67(\mathrm{MWCO}=5 \mathrm{kDa})$ and less than $0.1(\mathrm{MWCO}$ $=15 \mathrm{kDa}$ ). Moreover, Saurabh [2] published results for polymeric ultrafiltration membranes for a similar feed stream. The retention was about 0.7 for the membrane with $3000 \mathrm{Da} \mathrm{MWCO}$ and decreased to about 0.15 (MWCO $=20,000)$.

Schlesinger [3] tested different polymeric nanofiltration membranes for an analog application. The membranes NTR-7470 (Nitto-Denko), MPF-34 (Koch), NP030 (Microdyn Nadir) and Osmonics GE (Desal) showed retention higher than 0.90 for molecules with a mass higher than $1000 \mathrm{~g} \mathrm{~mol}^{-1}$. This is 
significantly higher compared to the ceramic ultrafiltration membranes however there is still a low retention for the gamma fraction. A more detailed study on the retention of different components has not been performed yet. Furthermore intense fouling was observed by Schlesinger [4,5].

In addition to the viscose-fiber production, membrane processes in strong alkaline solutions are under investigation for streams of the cotton industry as studied by Son [6]. An additional application, which is under investigation, is the treatment of caustic cleaning agents used in the dairy industry [7-9]. It has to be mentioned that the sodium hydroxide concentrations in these applications are much smaller than in the viscose-fiber industry.

There is much research work that describes the transport of small organic and inorganic molecules through nanofiltration membranes. Different research groups [10-13] published the influence of the $\mathrm{pH}$ value, the zeta potential and the hydrophobicity of the membrane as well as the charge of the molecule on the transport mechanism. Furthermore, Simon [14] studied the impact of caustic cleaning on the membrane performance. All this research was done in $\mathrm{pH}$ ranges from 2 to 12 , hence there is a lack of knowledge for higher $\mathrm{pH}$ values.

The influence of sodium hydroxide concentrations in the range of 1 up to $5 \mathrm{~mol} \mathrm{~L}^{-1}$ on the retention performance of nanofiltration membranes has not been studied so far. For several decades, highly alkaline stable nanofiltration membranes are available, although there are only few polymers available that can be used for such pH-robust membranes. One example is the membrane NP030 from Microdyn Nadir that is based on sulfonated polyethersulfone. Further, Advanced Membrane System Technologies supplies a highly alkaline stable nanofiltration membrane based on melamine polyamine.

Current research work is done to develop new membrane materials. Dalwani [15] developed a sulfonated polyether ether ketone (SPEEK)-based membrane with very high pH stability. Daems [16] developed a new technique to enhance the alkaline stability of PVDF membranes by grafting with polystyrene sulfonic acid.

Van Gestel [17] produced multilayer ceramic membranes with a MWCO lower than 200 Da. This was achieved with $\alpha-\mathrm{Al}_{2} \mathrm{O}_{3} / \gamma-\mathrm{Al}_{2} \mathrm{O}_{3} /$ anatase membranes that are stable in the $\mathrm{pH}$ range from 3 to 11 . At higher and lower $\mathrm{pH}$ values, the combination anatase on an $\alpha-\mathrm{Al}_{2} \mathrm{O}_{3}$ performs better.

In the present work, succinic acid was chosen as the model compound to investigate the retention behavior of gamma components due to several reasons. It has a molecular weight of $118 \mathrm{~g} \mathrm{~mol}^{-1}$, which is in the middle between acetic acid $\left(60 \mathrm{~g} \mathrm{~mol}^{-1}\right)$, and glucoisosaccharinic acid (180 $\left.\mathrm{g} \mathrm{mol}{ }^{-1}\right)$, two representative gamma components. Furthermore, its anion can act as mono- or divalent ion depending on the $\mathrm{pH}$ value.

A better understanding of the $\mathrm{NaOH}$ concentration impact on the retention behavior is important for the design of an efficient nanofiltration process, as partial dilution can improve the caustic recovery rate. The comparison of disodium succinate and sodium sulfate should help to improve the knowledge of membrane retention mechanisms and could indicate the possibility of separation between organic and inorganic divalent ions.

\section{Material and Methods}

\subsection{Material}

Succinic acid with a purity of $99.9 \%$ and disodium succinate with a purity of $99 \%$ were obtained from Sigma Aldrich. Hydroxide solution was prepared from deionized water and dry pellets with a purity of 99\% (Sigma Aldrich, Taufkirchen, Germany).

NP030 membrane samples from Microdyn Nadir (Wiesbaden, Germany) in DIN A4 format were purchased directly from the manufacturer and were used without any pretreatment. Membrane properties are shown in Table 1. 
Table 1. Overview of the membrane specifications found in the literature and on the material data sheet provided from the manufacturer.

\begin{tabular}{|c|c|c|}
\hline Parameter & Value & Source \\
\hline $\mathrm{Na}_{2} \mathrm{SO}_{4}$ retention ${ }^{1}[-]$ & $0.80-0.95$ & Microdyn Nadir \\
\hline $\mathrm{MWCO}(\mathrm{Da})$ & 520 & Kovac [18] \\
\hline MWCO (Da) & 400 & Boussu [19] \\
\hline Water permeance $\left[\mathrm{L} \mathrm{m}^{-2} \mathrm{~h}^{-1} \mathrm{bar}^{-1}\right]$ & 3.8 & Boussu [19] \\
\hline Water permeance $\left[\mathrm{L} \mathrm{m}^{-2} \mathrm{~h}^{-1} \mathrm{bar}^{-1}\right]$ & 4.4 & Bargeman [20] \\
\hline pH stability & $1-14$ & Microdyn Nadir \\
\hline Zeta potential pH $3(\mathrm{mV})$ & 1 & Boussu [19] \\
\hline Zeta potential pH $7(\mathrm{mV})$ & -15 & Boussu [19] \\
\hline Zeta potential pH $12(\mathrm{mV})$ & -20 & Boussu [19] \\
\hline Contac angle $\left(^{\circ}\right)$ & 88 & Boussu [19] \\
\hline Membrane material & permanently hydrophilic polyethersulfone & Microdyn Nadir \\
\hline
\end{tabular}

\subsection{Nanofiltration}

Experiments were performed on two different lab-scale nanofiltration rigs. One was a modified OSMO-MC-01 flat-sheet lab-scale device from OSMOTA (Rutesheim, Germany), the other one was a MEMCELL 3 from OSMO (Korntal-Münchingen, Germany). A heat exchanger was added in the feed stream of both systems for temperature regulation. The membrane area was $80 \mathrm{~cm}^{2}$; for the OSMO-MC-01 and three times $80 \mathrm{~cm}^{2}$ in the MEMCELL 3 device. The cells were arranged in parallel. Permeate and retentate were returned to the feed tank to keep the feed concentration constant. The samples amount was kept low $(\sim 1 \mathrm{~mL})$ compared to the feed volume $(5 \mathrm{~L})$. The feed concentration was measured throughout the experiment to ensure stable concentrations.

The refractive index of permeate was observed to identify if a quasi-stationary state was achieved. Samples of at least six different pressures were collected with each combination of parameters.

All experiments at a certain $\mathrm{NaOH}$ concentration were performed with the same membrane. Before the experiment, the membrane was rinsed with a $\mathrm{NaOH}$ solution until constant permeate flux (approx. 50-80 h). The $\mathrm{NaOH}$ concentration during this pretreatment was equal to the concentration in the following experiment.

\subsection{Refractometer}

Refractive index measurements were performed with the refractometer Abbemat 500 from Anton Paar (Graz, Austria). The refractometer was used on the one hand to check constant permeate composition and on the other hand to monitor constant feed composition.

Furthermore, the refractive index measurements were used to measure the $\mathrm{NaOH}$ concentration.

Calibration data were measured for different solutions of succinic acid $\left(0-0.85 \mathrm{~mol} \mathrm{~L}^{-1}\right)$ in different $\mathrm{NaOH}$ concentrations $\left(0-5 \mathrm{~mol} \mathrm{~L}^{-1}\right)$. The correlation of the refractive index $(n)$ based on $\mathrm{NaOH}$ concentration $\left(c_{\mathrm{NaOH}}\right.$ in mol L$\left.{ }^{-1}\right)$ and succinic acid concentration $\left(c_{S A}\right.$ in $\left.\mathrm{mol} \mathrm{L}^{-1}\right)$ is given by Equation (1).

$$
n=A+B \times c_{\mathrm{NaOH}}+C \times c_{S A}+D \times c_{\mathrm{NaOH}} \times c_{S A}+E \times c_{\mathrm{NaOH}}^{2}+F \times c_{S A}^{2}
$$

where $A=1.333350 ; B=0.010348 ; C=0.008236 ; D=-0.001227 ; E=-0.000337 ; F=-0.000284$.

Based on this correlation, the $\mathrm{NaOH}$ concentration can be calculated for a given succinic acid concentration.

\subsection{Titration}

To verify the calculated $\mathrm{NaOH}$ concentration (based on the refractive index), a batch of samples was additionally analyzed by titration with $0.25 \mathrm{~mol} \mathrm{~L}^{-1}$ sulfuric acid to $\mathrm{pH} 3.7$. 
To eliminate the influence of the titrant, preliminary tests were done with sulfuric acid and hydrochloric acid. No significant influence of the titrant was observed.

Furthermore, preliminary tests showed that succinic acid is completely undissociated at pH 3.7. This needs to be considered for the calculation of the sodium retention based on titration.

\subsection{Chromatography}

Succinic acid and disodium succinate were quantified by high performance liquid chromatography (HPLC) analysis. A Thermo Hypersil GOLD aQ $250 \mathrm{~mm}$ column with the photodiode array detector Dionex UVD 340U was used. A wavelength of $210 \mathrm{~nm}$ was applied for detection. Sulfate concentration was analyzed using ion chromatography. A CarboPac PA10 was used as a column. The detector for quantification was a Dionex ED 50. All the HPLC instruments used were purchased from Thermo Fisher Scientific (Vienna, Austria). The data processing was done with the software Chromeleon (version 7.2).

\subsection{Scanning Electron Microscopy}

SEM measurements were done for different membranes before and after the experiments. The used membrane samples were washed with deionized water and subsequently dried. The unused membrane sample was used as received from the manufacturer.

The samples were gold-plated prior to the analysis. Images were taken using a FEI Quanta 450 with $10 \mathrm{Kv}$ Thermo Fisher Scientific (Vienna, Austria). For the image processing the software Phenom ProSuite (version 2.9.0.0) was used.

\subsection{Attenuated Total Reflectance Infrared Spectroscopy}

All analyzed membrane samples except the unused one were washed with deionized water for one minute after exposure to $\mathrm{NaOH}$ solution.

Measurements were done with a Bruker Tensor 27 Spectrometer, which was equipped with a Specac Single Reflection Diamond ATR “Golden Gate" (Bruker Austria GmbH, Vienna, Austria). The software OPUS (version 7.5). was used for data processing.

\section{Theoretical Background}

Two different models were used to assess experimental data.

\subsection{Spiegler and Kedem Model}

The Spiegler and Kedem model [21] is based on the thermodynamics of irreversible processes. The flux of the solvent $J_{L}$ is defined by:

$$
J_{L}=-P_{L} \times\left(\frac{d p}{d z}-\sigma \times \frac{d \Pi}{d z}\right)
$$

where $P_{L}$ is the permeability of solvent, $d p / d z$ the pressure gradient through the membrane and $d \pi / d z$ the osmotic pressure gradient. $\sigma$ represents the reflection coefficient.

The flux of the solute $J_{S}$ is given by:

$$
J_{S}=-P_{S} \times \frac{d c_{S}}{d z}+(1-\sigma) \times c_{S} \times J_{L}
$$

$P_{S}$ is the permeability of the solute, $d c_{S} / d z$ the concentration gradient and $c_{S}$ the concentration of solute. The first term is related to transport due to diffusion and the second one represents convective 
transport. If $\sigma=1$ the Spiegler and Kedem model conforms to the solution-diffusion model. With the common definition of the retention $R$, as expressed in Equation (4),

$$
R=1-\frac{c_{P e r m}}{c_{\text {Ret }}}
$$

and further calculations, the retention can be written as a function of the overall permeate flux $J_{V}$ and the two model coefficients $\sigma$ and $P$ :

$$
R=1-\frac{1-\sigma}{1-\sigma \times \exp \left(\frac{(\sigma-1) \times J_{V}}{P}\right)}
$$

with:

$$
P=\frac{P_{S}}{z}
$$

By fitting the experimental data, $\sigma$ and $P$ can be evaluated for any combination of temperature and concentrations.

\subsection{Pusch Model}

The Pusch model is another well-known model, which can also be used to describe the nanofiltration processes. It is based on thermodynamics of irreversible processes and was expounded by Pusch in 1977 [22]. The equation describes the reciprocal value of the retention $(R)$ as a function of the reciprocal retention at infinite flux $\left(R_{\infty}\right)$, two constants $\left(L_{D}, L_{P}\right)$, the osmotic pressure difference $(\pi)$ and the reciprocal value of the flux $\left(J_{V}\right)$.

$$
\frac{1}{R}=\frac{1}{R_{\infty}}+\left(\frac{L_{D}}{L_{P}}-R_{\infty}{ }^{2}\right) \times \frac{L_{P} \times \Pi_{1}}{R_{\infty} \times J_{V}}
$$

To calculate $R_{\infty}$ the reciprocal value of the flux is plotted on the x-axis and the reciprocal value of the retention on the y-axis. A linear correlation of this set of data points gives the reciprocal values of $R_{\infty}$ as the ordinate intercept.

\subsection{Law of Electroneutrality}

Due to the law of electroneutrality, the number of negatively charged groups must be equal to the number of positively charged groups. Succinic acid completely dissociates in highly alkaline solutions. Consequently, the succinate has two negatively charged groups per molecule. Due to the absence of other cations, the sodium ions equalize the negative charge of the succinate. Because of this, the retention of sodium is related to the retention of the succinate as shown here:

$$
c_{R, N a} \times R_{N a}=c_{R, S u} \times R_{S u} \times 2
$$

$c_{R, N a}$ and $c_{R, S u}$ represent the concentration of sodium and succinate in the retentate. $R_{N a}$ denotes the retention of sodium and $R_{S u}$ indicates the retention of succinate. Equation (8) applies only to strongly alkaline solutions in which the amount of hydronium ions is negligible and thus sodium is the only cation in the solution.

\section{Results and Discussion}

\subsection{Saturation Solubility of Succinic Acid}

The saturation solubility of succinic acid increases with increasing concentration of $\mathrm{NaOH}$ as shown in Figure 1. The solubility in $5 \mathrm{M} \mathrm{NaOH}$ is four times higher than in water. Succinic acid in protonated form is less soluble than the salt form. An increasing $\mathrm{NaOH}$ concentration enhances the dissociation and therefore the solubility. Solubility at $40{ }^{\circ} \mathrm{C}$ is on average $0.24 \mathrm{~mol} \mathrm{~L}-1$ higher than at 30 
${ }^{\circ} \mathrm{C}$. The solubility of disodium succinate in water is approximately twice as high as that of succinic acid. Thus, the succinic acid and disodium succinate concentrations used in the nanofiltration experiments were far below the solubility limits.

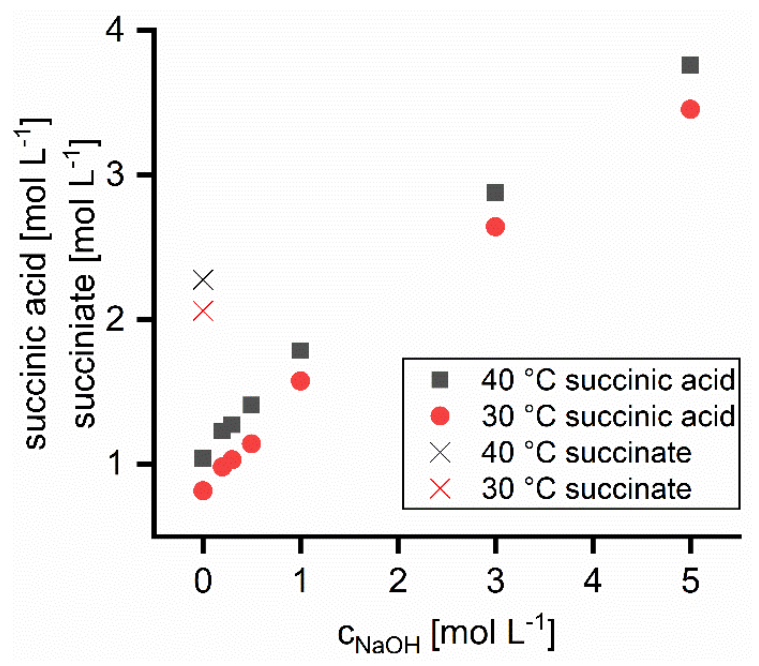

Figure 1. Saturation concentration of succinic acid in $\mathrm{NaOH}$ solutions at 30 and $40^{\circ} \mathrm{C}$ (dots). Crosses mark the saturation solubility of disodium succinate in water.

\subsection{Flux}

Figure 2 shows the permeate flux of water and $\mathrm{NaOH}$ solutions with different concentrations. As can be seen, the flux is linearly dependent on the applied pressure in the range of 5 to 30 bar.

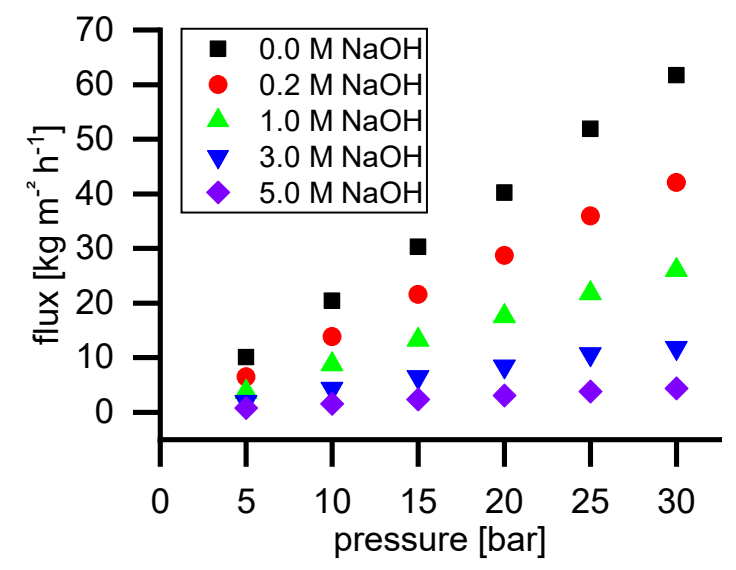

Figure 2. Permeate flux of water and $\mathrm{NaOH}$ solutions with different concentrations in the range from 5 to 30 bar at $40{ }^{\circ} \mathrm{C}$.

Figure 3 shows the influence of the $\mathrm{NaOH}$ concentration on the permeability coefficient $\left(L_{P}\right)$ at $40{ }^{\circ} \mathrm{C}$. By increasing the $\mathrm{NaOH}$ concentration from 0 to $5 \mathrm{~mol}, \mathrm{~L}^{-1} L_{P}$ decreases from 2.05 to $0.15 \mathrm{~kg} \mathrm{~m}^{-2} \mathrm{~h}^{-1} \mathrm{bar}^{-1}$. The flux decrease by increasing $\mathrm{NaOH}$ concentration occurs for two different reasons. Firstly, the viscosity increases from $0.65 \mathrm{mPa} \cdot \mathrm{s}$ (water) to $2.5 \mathrm{mPa} \cdot \mathrm{s}(5 \mathrm{M} \mathrm{NaOH})$. The second reason is the difference in osmotic pressure. The NP030 shows a small retention for $\mathrm{NaOH}$. Consequently, an osmotic pressure difference occurs leading to a reduction of permeate flux. The higher the feed concentration, the higher is the osmotic pressure difference. 


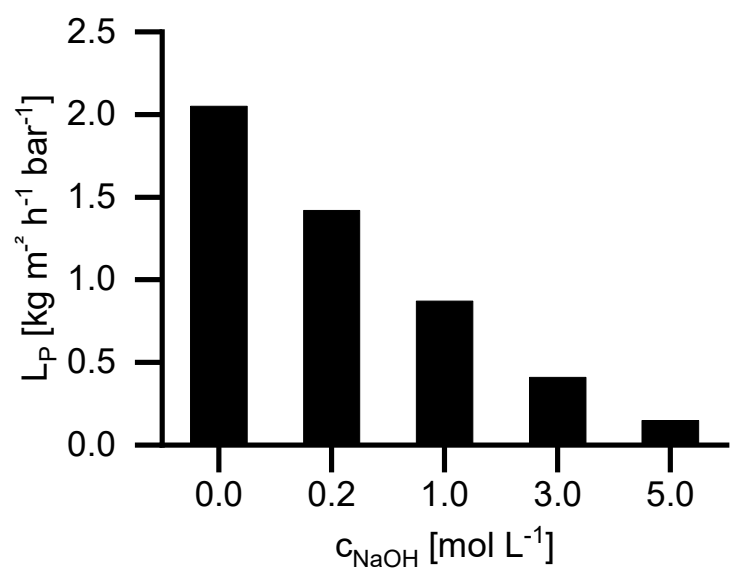

Figure 3. Permeability coefficient $\left(L_{P}\right)$ of water and $\mathrm{NaOH}$ solutions of different concentrations at $40{ }^{\circ} \mathrm{C}$.

\subsection{Retention of Sodium Sulfate}

The retention value of sulfate, according to manufacturer data, is between 0.80 and 0.95 . The experiments were performed with 40 bar at $20^{\circ} \mathrm{C}$, details on the feed concentration were not given. Sulfate retention was studied to compare the influence of the structure of two different divalent ions. The organic succinate ion has an oval structure with the negatively charged groups on both ends. In contrast, the sulfate ion is circular. Figure 4 shows the retention of sulfate with a feed concentration of $0.1 \mathrm{~mol} \mathrm{~L}^{-1}$ sodium sulfate. As can be seen in the performed experiments, the retention of sulfate in aqueous solution was lower than 0.8 for permeate fluxes lower than $30 \mathrm{~kg} \mathrm{~m}^{-2} \mathrm{~h}^{-1}$. At higher fluxes, the sulfate retention was in the range specified by the supplier. The reflection coefficient found in the Spiegler and Kedem model was calculated to be 0.9 .

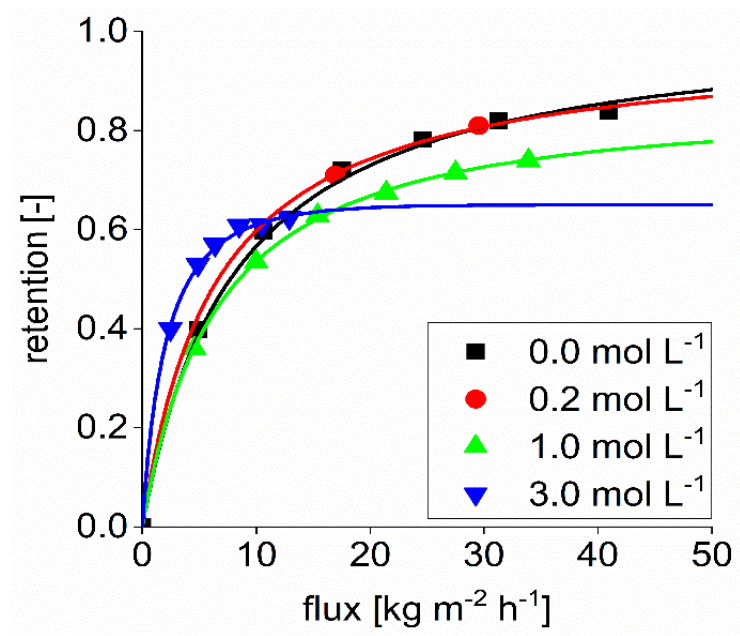

Figure 4. Retention of sulfate in pure water and in mixtures of different $\mathrm{NaOH}$ concentrations at $40{ }^{\circ} \mathrm{C}$. The dots indicate experimental data, the solid line shows the Spiegler and Kedem fit.

Furthermore, it can be seen that the sulfate retention is reduced by adding $\mathrm{NaOH}$ to the solution. The higher the $\mathrm{NaOH}$ concentration is, the lower the sulfate retention.

\subsection{Retention of Succinic Acid}

Figure 5 shows the retention at infinite permeate flux calculated with the Pusch model $\left(R_{\infty}\right)$ in dependency of the temperature, the feed concentration of succinic acid and the $\mathrm{NaOH}$ concentration. Figure 6 shows the reflection coefficient $\left(\sigma_{S A}\right)$ of the Spiegler and Kedem model with the same parameters. The standard deviation for the Spiegler and Kedem fit is significantly smaller than for 
the Pusch Model. Consequently, the $R_{\infty}$ values show a higher variance than the $\sigma_{S A}$ values. Hence, further calculations were performed only with the Spiegler and Kedem model.

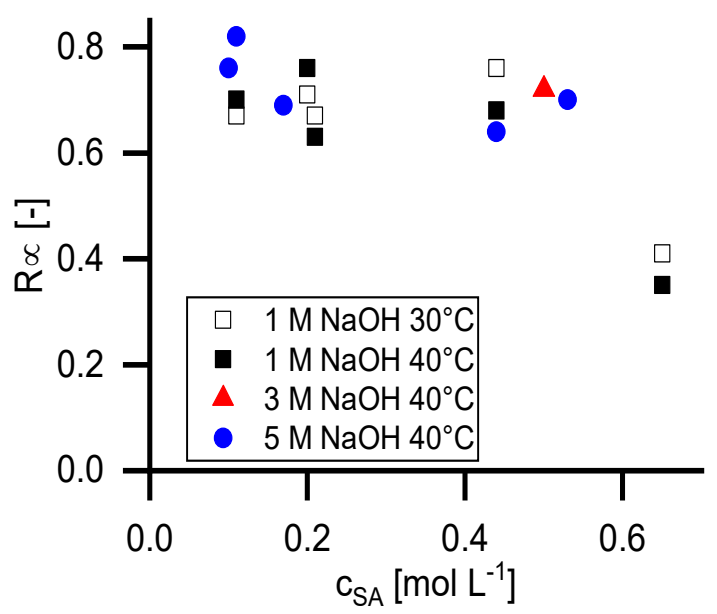

Figure 5. Retention of succinic acid at infinite permeate flux $\left(R_{\infty}\right)$ in dependency of feed concentration calculated with the Pusch model. $\mathrm{NaOH}$ concentration and temperature are shown as process parameters for the generated data.

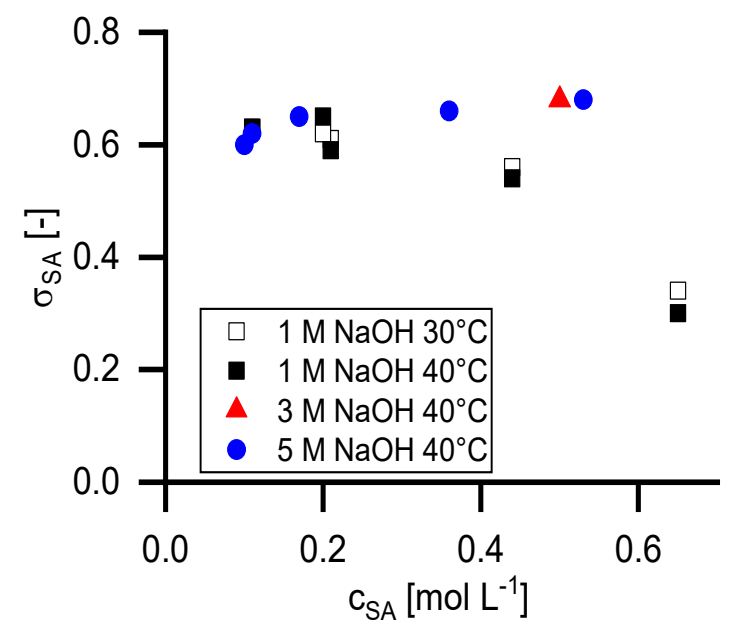

Figure 6. Reflection coefficients of succinic acid in dependency of feed concentration according the Spiegler and Kedem model.

As it is shown in Figure 6, the reflection coefficient of succinic acid is independent from the $\mathrm{NaOH}$ concentration as long as the succinate ion is completely dissociated. The incompletely dissociated form shows significantly lower $\sigma_{S A}$ values. The $\sigma_{S A}$ values at $0.43 \mathrm{~mol} \mathrm{~L}^{-1}$ succinic acid in $1 \mathrm{molar} \mathrm{NaOH}$ solution are lower compared to the data points at complete dissociation.

The experiments with $0.2 \mathrm{~mol} \mathrm{~L}^{-1}$ succinic acid were performed twice, once as a first experiment of a series and a second time at the end of a series. As shown, the results have a good reproducibility.

The succinate ion shows a similar retention in 0.1 molar aqueous disodium succinate solution (chapter 4.6.) as sulfate in a 0.1 molar sodium sulfate solution. The situation changes for the sulfate ion in a caustic solution. Its retention decreases with increasing $\mathrm{NaOH}$ concentration. In comparison, the succinate retention is unaffected from $\mathrm{NaOH}$ in a wide concentration range. A difference in the capability of hydration shell formation can explain this. It seems that the succinate ion is able to pull the water molecules stronger than the sulfate ion. Bouchoux [23] pointed out, that the sodium lactate retention is almost unaffected by adding sodium chloride to the solution, in contrast glucose retention decreased significantly. This means organic anions like lactate and succinate are able to form hydration shells, which are less affected from competitive hydration of other ions in the solution. Hence, the type 
and concentration of co-ions in the solution can control the separation performance of ions. To prove this assumption, further investigations with molecules with different structures are necessary.

In the case of incomplete dissociation of the succinic acid, the retention decreases by about half. This is within the same range as the chloride retention specified by the manufacturer $(0.25-0.35)$.

\subsection{Retention of Sodium Ions}

Figure 7 shows the reflection coefficient of sodium ions $\left(\sigma_{N a}\right)$. As mentioned in Equation (8) the retention of sodium ions is related to the retention of succinate ions by the law of electroneutrality, assuming that no further cations are present. In solutions containing excess $\mathrm{NaOH}$ with respect to the amount of carboxylic acid groups, this requirement is met. According to Equation (8) the slope of the data points for $1 \mathrm{M} \mathrm{NaOH}$ is five times steeper than for $5 \mathrm{M} \mathrm{NaOH}$. The calculated slopes are $1.09(1 \mathrm{M}$ $\mathrm{NaOH})$ and $0.22(5 \mathrm{M} \mathrm{NaOH})$. The decrease of the $\mathrm{pH}$ value leads to the presence of hydronium ions, which decouples the retention between the sodium and succinate ions. Thus, this point was not taken into account for the slope calculation.

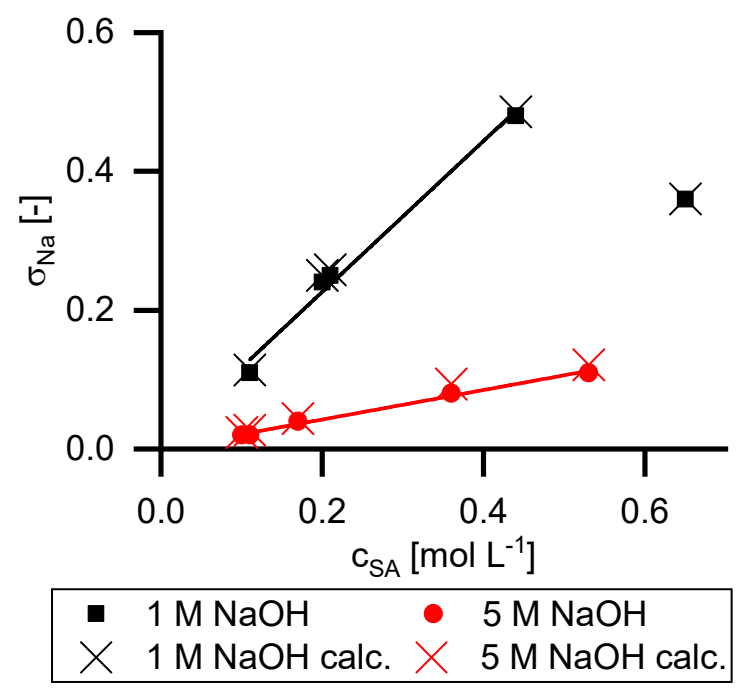

Figure 7. Reflection coefficients of the sodium ions in dependency of succinic acid feed concentration. The dots represent calculated values with the Spiegler and Kedem model, based on experimental data. Crosses represent the calculated reflection coefficients according to Equation (8).

Additionally, the retention of sodium ions was calculated with Equation (8). The results are given as crosses in Figure 7. As can be seen, the experimental data and the calculated values are in good agreement.

\subsection{Retention of Disodium Succinate}

To investigate the retention of equimolar solutions of sodium ions and carboxylic groups, experiments with disodium succinate were performed. Figure 8 shows the reflection coefficient in relation to feed concentrations. As can be seen, the retention for disodium succinate in water is higher than the retention of succinic acid in $\mathrm{NaOH}$ solutions. Furthermore, it can be seen, that the reflection coefficient decreases with increasing feed concentration. 


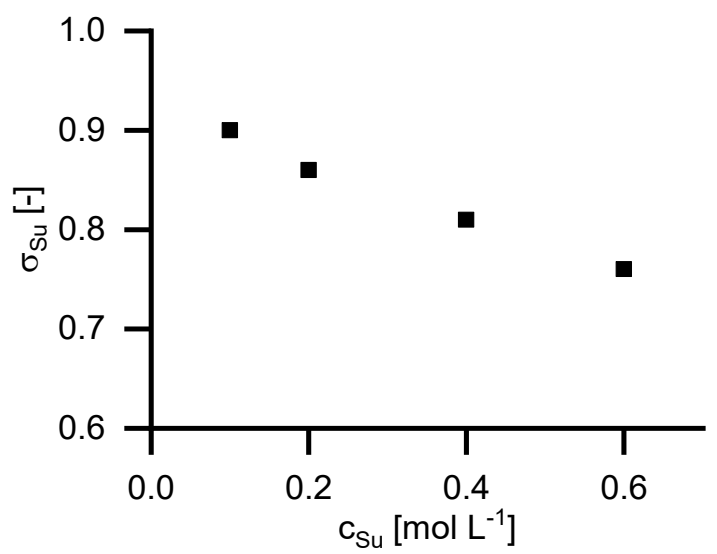

Figure 8. Reflection coefficient of disodium succinate in aqueous solution at $40{ }^{\circ} \mathrm{C}$ in relation to the feed concentration of succinate.

\subsection{SEM Measurements}

Rinsing the membrane with water leads to a tripling of the active membrane layer thickness due to a swelling effect. Swelling in caustic solution leads to the same result (Figure 9).

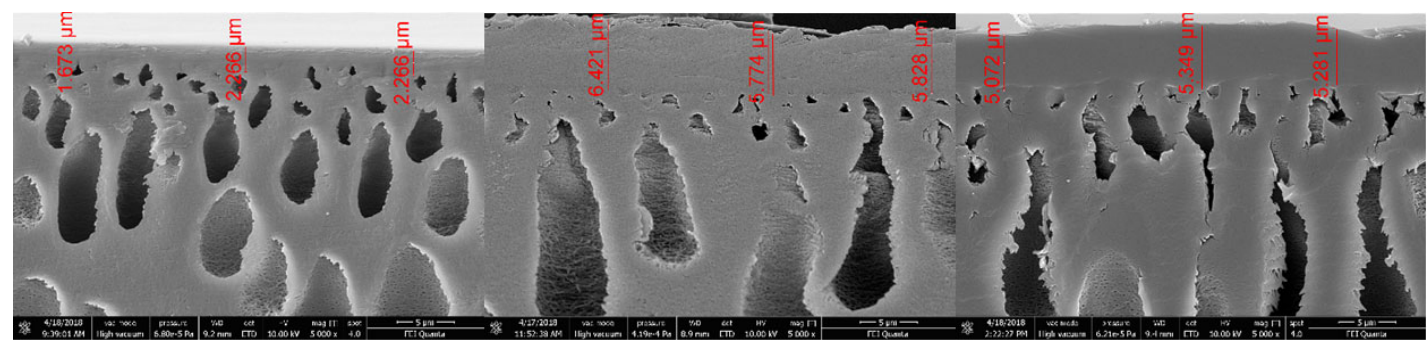

Figure 9. SEM measurements of a new membrane (left), a pre-conditioned membrane (middle), and a used membrane at $40{ }^{\circ} \mathrm{C}$ and 25 bar (right). The shown values represent the active membrane layer thickness.

No changes of the active layer thickness can be seen by samples of the used membranes stressed up to 25 bar at $40{ }^{\circ} \mathrm{C}$. The shape of the pores in the first support layer changes from an oval shape to an elongated shape. This does not influence the overall membrane performance.

By increasing the pressure up to $55 \mathrm{bar}\left(40^{\circ} \mathrm{C}\right)$, a significant change can be observed, see Figure 10. The small pores of the first support layer disappear and a compact layer is formed. The compression of this support layer is irreversible and leads to a significantly lower permeate flux. The bigger pores in the bottom part of the first support layer are still present.

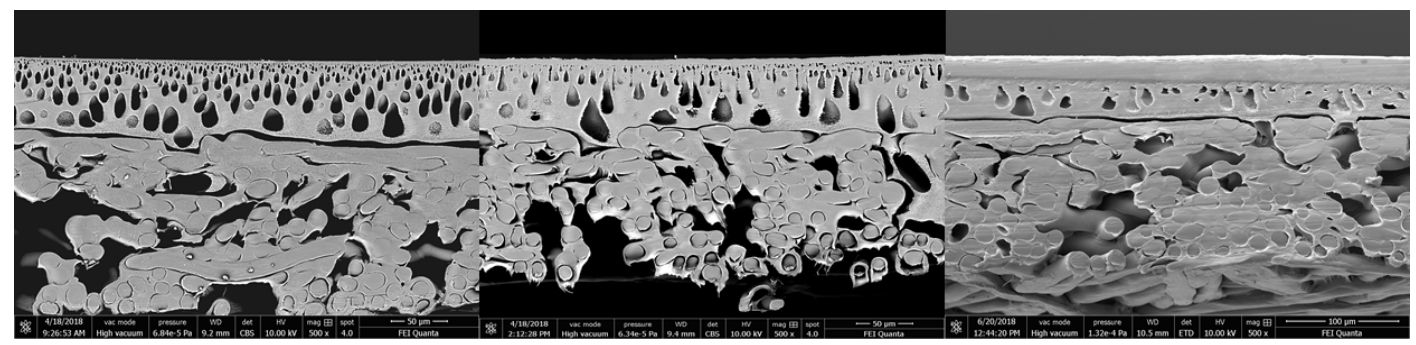

Figure 10. SEM measurement of the whole cross-sections of an unused membrane (left), a lowly stressed (max. 25 bar) membrane (middle), and a highly stressed (max. 55 bar membrane) (right).

\subsection{ATR-IR Measurement}

Figure 11 shows ATR-IR measurements of different conditioned membranes. 


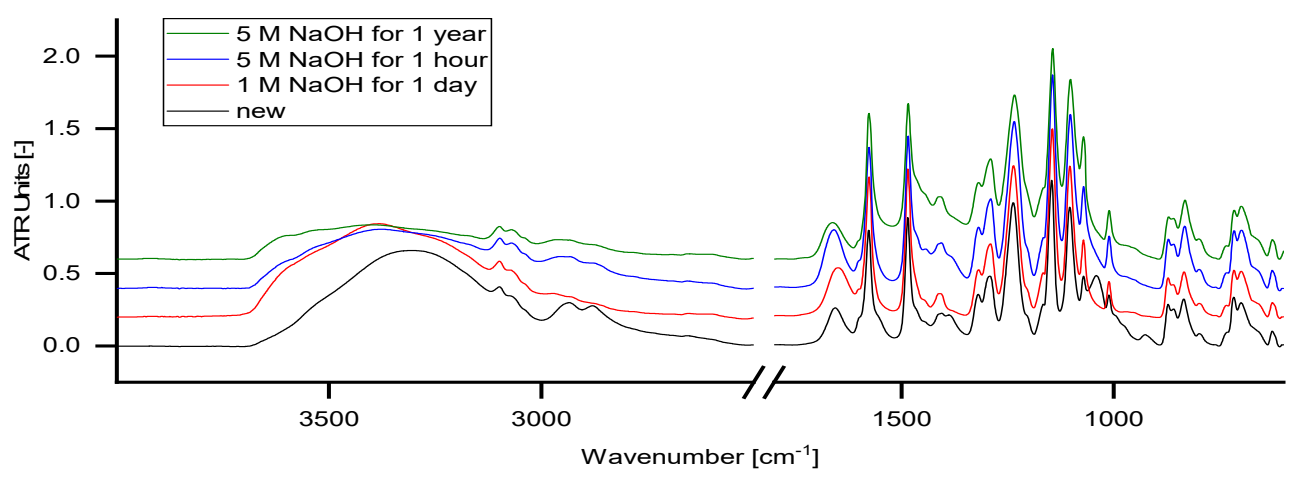

Figure 11. ATR-IR spectra of membranes stored in $\mathrm{NaOH}$ solutions with different concentrations (1-5 molar) for varying periods ( $1 \mathrm{~h}-1$ year).

Essentially, the spectra of all tested membranes resemble the spectrum of PES [Poly(p-phenylene ether sulfone)] which is declared as the main membrane polymer by the supplier.

The band at a wavelength from $3200-3500 \mathrm{~cm}^{-1}$ represents the $\mathrm{O}-\mathrm{H}$ stretching band. In contact with $\mathrm{NaOH}$ solution, a change in the ATR-IR diagram can be observed but is not related to any changes in the membrane polymer composition. These changes appear within the first hour and do not alter significantly over time.

The bands at 2934 and $2879 \mathrm{~cm}^{-1}$ represent aliphatic C-H stretching bonds which decrease in contact with $\mathrm{NaOH}$ solution. The bands at 1040 and $925 \mathrm{~cm}^{-1}$ refer to glycerol, which was used for membrane preparation or storage and is washed out immediately by the solutions. The band at $1400 \mathrm{~cm}^{-1}$ indicates decompositions of carbonates on the membrane. Carbonates were formed from carbon dioxide, which is dissolved in the solution. By longer exposure to the $\mathrm{NaOH}$ solution, the band increases slightly. None of the characteristic polymer bands changes significantly with the treatment time. Consequently, NP030 can be considered as chemically stable in alkaline solutions with concentrations up to $5 \mathrm{~mol} \mathrm{~L}^{-1} \mathrm{NaOH}$. This is in good agreement with the data published by Schlesinger [3], which indicates high alkali resistance of the NP030.

\section{Conclusions}

Based on the retention results of aqueous solutions, it can be concluded that the organic salt (disodium succinate) behaves similarly to the inorganic sodium sulfate. A different retention behavior is observed in $\mathrm{NaOH}$ solutions. Sulfate ions are more affected by the presence of co-ions than succinate ions. Degree of dissociation is the factor with the highest impact on the retention of succinic acid. Consequently, the charge selectivity of the NP030 nanofiltration membrane could be verified with an organic salt by changing its degree of dissociation. A higher $\mathrm{NaOH}$ concentration in the feed solution leads to a significant decrease of permeate flux.

The retention of completely dissociated succinate, is unaffected by the $\mathrm{NaOH}$ concentration. Hence, a dilution during the nanofiltration process may improve the process efficiency, as the flux increases. Furthermore, the selectivity between sulfate and succinate can be controlled by the $\mathrm{NaOH}$ concentration.

The results of the ATR-IR measurements and the thermomechanical stress tests indicate an applicability of the NP030 membrane in highly alkaline conditions. Further performance tests with model solutions are necessary to prove this.

Furthermore, it is shown, that the Spiegler and Kedem model appropriately describes the retention behavior of all investigated species, even in five molar $\mathrm{NaOH}$.

Author Contributions: Conceptualization, K.S.; Investigation, K.S.; Project administration, R.H.; Supervision, W.S.; Writing—original draft, K.S.; Writing—review \& editing, W.S.

Funding: This research was funded by the Austrian Research Promotion Agency (FFG), grant number 844608 . The APC was funded by Kompetentzzentrum Holz GmbH. 
Acknowledgments: Financial support was provided by the Austrian government, the provinces of Lower Austria, Upper Austria, and Carinthia as well as by Lenzing AG. We also express our gratitude to the Johannes Kepler University, Linz, the University of Natural Resources and Life Sciences (BOKU), Vienna, and Lenzing AG for their in-kind contributions. Furthermore, we would like to thank the responsible project manager at Lenzing AG Robert Bischof. Another big thank you goes to Erwin Malzner and his team for the extraordinary support in the laboratory of Lenzing AG.

Conflicts of Interest: The authors declare no conflict of interest.

\section{References}

1. Singh, S.C.; Khare, R.A. Effect of hemicelluloses on pulp characteristics and use of ceramic membranes in the separation of hemicelluloses from highly alkaline industrial process stream. Cellulose 2018, 25, 2561-2572. [CrossRef]

2. Singh, S.C.; Murthy, Z.V.P. Hemicelluloses separation from caustic-containing process stream by ultrafiltration. Sep. Sci. Technol. 2017, 52, 2252-2261. [CrossRef]

3. Schlesinger, R.; Götzinger, G.; Sixta, H.; Friedl, A.; Harasek, M. Evaluation of alkali resistant nanofiltration membranes for the separation of hemicellulose from concentrated alkaline process liquors. Desalination 2006, 192, 303-314. [CrossRef]

4. Schlesinger, R.; Röder, T.; Götzinger, G.; Sixta, H.; Harasek, M.; Friedl, A. Influence of hemicellulose aggregate and gel layer formation on flux and retention during nanofiltration of alkaline solutions. Desalination 2005, 175, 121-134. [CrossRef]

5. Schlesinger, R.; Röder, T.; Götzinger, G.; Harasek, M.; Sixta, H.; Weber, H. Hemicellulose fouling behaviour during nanofiltration of press liquor. Lenzing. Ber. 2004, 83, 46-54.

6. Son, E.J.; Choe, E.K.; Kim, J.W. Nanofiltration membrane technology for caustic soda recovery. Text. Chem. Color. Am. Dyest. Report. 2000, 32, 46-52.

7. Gesan-Guiziou, G.; Boyaval, E.; Daufin, G. Nanofiltration for the recovery of caustic cleaning-in-place solutions: Robustness towards large variations of composition. J. Dairy Res. 2002, 69, 633-643. [CrossRef] [PubMed]

8. Trägårdh, G.; Johansson, D. Purification of alkaline cleaning solutions from the dairy industry using membrane separation technology. Desalination 1998, 119, 21-29. [CrossRef]

9. Gesan-guiziou, G.; Alvarez, N.; Jacob, D.; Daufin, G. Cleaning-in-place coupled with membrane regeneration for re-using caustic soda solutions. Sep. Purif. Technol. 2007, 54, 329-339. [CrossRef]

10. Braeken, L.; Bettens, B.; Boussu, K.; van der Meeren, P.; Cocquyt, J.; Vermant, J.; van der Bruggen, B. Transport mechanisms of dissolved organic compounds in aqueous solution during nanofiltration. J. Membr. Sci. 2006, 279, 311-319. [CrossRef]

11. Luo, J.; Ding, L. Influence of $\mathrm{pH}$ on treatment of dairy wastewater by nanofiltration using shear-enhanced filtration system. Desalination 2011, 278, 150-156. [CrossRef]

12. Mänttäri, M.; Pihlajamäki, A.; Nyström, M. Effect of $\mathrm{pH}$ on hydrophilicity and charge and their effect on the filtration efficiency of NF membranes at different pH. J. Membr. Sci. 2006, 280, 311-320. [CrossRef]

13. Dalwani, M.; Benes, N.E.; Bargeman, G.; Stamatialis, D.; Wessling, M. A method for characterizing membranes during nanofiltration at extreme pH. J. Membr. Sci. 2010, 363, 188-194. [CrossRef]

14. Simon, A.; Mcdonald, J.A.; Khan, S.J.; Price, W.E.; Nghiem, L.D. Effects of caustic cleaning on pore size of nanofiltration membranes and their rejection of trace organic chemicals. J. Membr. Sci. 2013, 447, 153-162. [CrossRef]

15. Dalwani, M.; Bargeman, G.; Schwan, S.; Boerrigter, M.; Wessling, M.; Benes, N.E. Sulfonated poly (ether ether ketone) based composite membranes for nanofiltration of acidic and alkaline media. J. Membr. Sci. 2011, 381, 81-89. [CrossRef]

16. Daems, N.; Milis, A.S.S.; Verbeke, R.; Pescarmona, P.P.; Vankelecom, I.F.J. High-performance membranes with full pH-stability. R. Soc. Chem. Adv. 2018, 8, 8813-8827. [CrossRef]

17. van Gestel, T.; Vandecasteele, C.; Buekenhoudt, A.; Dotremont, C.; Luyten, J.; Leysen, R.; van der Bruggen, B.; Maes, G. Alumina and titania multilayer membranes for nanofiltration: Preparation, characterization and chemical stability. J. Membr. Sci. 2002, 207, 73-89. [CrossRef]

18. Kovac, Z.; Samhaber, W. Characterization of nanofiltration membranes with uncharged solutes. Membrántechnika 2008, 12, 22-36. 
19. Boussu, K.; van der Bruggen, B.; Volodin, A.; van Haesendonck, C. Characterization of commercial nanofiltration membranes and comparison with self-made polyethersulfone membranes. Desalination 2006, 191, 245-253. [CrossRef]

20. Bargeman, G.; Westerink, J.B.; Miguez, O.G.; Wessling, M. The effect of $\mathrm{NaCl}$ and glucose concentration on retentions for nanofiltration membranes processing concentrated solutions. Sep. Purif. Technol. 2014, 134, 46-57. [CrossRef]

21. Spiegler, K.S.; Kedem, O. Thermodynamics of hyperfiltration (reverse osmosis): Criteria for efficient membranes. Desalination 1966, 1, 311-326. [CrossRef]

22. Pusch, W. Determination of Transport Parameters of Synthetic Membranes by Hyperfiltration Experiments Part I: Derivation of Transport Relationship from the Linear Relations of Thermodynamics of Irreversible Processes. Ber. Der Bunsenges. Für Phys. Chem. 1977, 81, 269-276. [CrossRef]

23. Bouchoux, A.; Balmann, R.; Lutin, F. Nanofiltration of glucose and sodium lactate solutions Variations of retention between single-and mixed-solute solutions. J. Membr. Sci. 2005, 258, 123-132. [CrossRef]

(C) 2019 by the authors. Licensee MDPI, Basel, Switzerland. This article is an open access article distributed under the terms and conditions of the Creative Commons Attribution (CC BY) license (http://creativecommons.org/licenses/by/4.0/). 